\title{
Onset of transverse instabilities of confined dark solitons
}

\author{
M. A. Hoefer ${ }^{1}$ and B. Ilan $^{2}$ \\ ${ }^{1}$ Department of Applied Mathematics, University of Colorado, Boulder, CO 80309, USA* \\ ${ }^{2}$ School of Natural Sciences, University of California-Merced, Merced, CA, 95343, USA ${ }^{\dagger}$
}

(Dated: March 16, 2022)

\begin{abstract}
We investigate propagating dark soliton solutions of the two-dimensional defocusing nonlinear Schrödinger / Gross-Pitaevskii (NLS/GP) equation that are transversely confined to propagate in an infinitely long channel. Families of single, vortex, and multi-lobed solitons are computed using a spectrally-accurate numerical scheme. The multi-lobed solitons are unstable to small transverse perturbations. However, the single-lobed solitons are stable if they are sufficiently confined along the transverse direction, which explains their effective one-dimensional dynamics. The emergence of a transverse modulational instability is characterized in terms of a spectral bifurcation. The critical confinement width for this bifurcation is found to coincide with the existence of a propagating vortex solution and the onset of a "snaking" instability in the dark soliton dynamics that, in turn, give rise to vortex or multi-vortex excitations. These results shed light on the superfluidic hydrodynamics of dispersive shock waves in Bose-Einstein condensates and nonlinear optics.
\end{abstract}

Solitary waves are ubiquitous in nonlinear dispersive systems. Isolated solitary waves are often either elevation or depression waves, also called bright or dark solitons, respectively. Dark solitons, also known as kink solitons, require a non vanishing background and their mathematical study has been extensive (cf. [1-3]). Experimentally, temporal and spatial dark solitons have been observed in optical fibers [4-6] and waveguide arrays [7], surface water waves [8, 9], plasmas [10], and Bose-Einstein condensates (BECs) [11, 12]. Dark solitons are one-dimensional objects that can naturally be extended to higher dimensions as planar dark solitons. In experiments, the transverse direction is spatially confined. For example, by use of an appropriately shaped electromagnetic potential, a BEC can be confined into a "pancake" or "cigar" shape, yielding effective two-dimensional or one-dimensional dynamics, respectively. Moreover, it has been observed experimentally that these effectively one-dimensional BEC dynamics are stable when sufficiently confined $[11,12]$ and unstable otherwise [13,14]. This raises the question: what is the threshold confinement width for effectively lower-dimensional dynamics of dark solitons in nonlinear wave systems?

To address this question, we consider the mean field, superfluidic (dissipationless) dynamics of a BEC governed by the $(2+1)$-dimensional defocusing nonlinear Schrödinger / Gross-Pitaveskii (NLS/GP) equation. In one dimension, this equation admits dark soliton solutions. In multiple dimensions, the NLS/GP equation admits line or planar dark solitons, which are uniform along all but the direction of propagation. Exact propagating line dark solitons solutions can be written explicitly and their stability has been analyzed extensively. In particular, they are modulationally unstable to small transverse perturbations [15].

\footnotetext{
* hoefer@colorado.edu

† bilan@ucmerced.edu
}

Previous studies have obtained critical confinement thresholds for stationary or "black" solitons $[16,17]$ and propagating or "gray" solitons [18]. In the latter case for gray solitons, the stability of approximate soliton solutions of a $(3+1)$-dimensional GP equation with transverse confinement achieved by a harmonic potential were obtained as follows. An initial black soliton was dynamically evolved in the presence of dissipation, leading to a reduction in amplitude and an approximate confined gray soliton. These solutions were then used to linearize the GP equation and the resulting equations were evolved numerically and analyzed for amplitude growth (instability).

In our study, the critical confinement width for $(2+1)$ dimensional propagating dark solitons is computed and found to occur at the onset of a transverse instability. Specifically, generalized bound state (or solitary wave) solutions are computed in a two-dimensional channel, which is confined along the $y$ axis and unbounded along the $x$ axis. Along the confined direction, either Dirichlet (impenetrable wall) or Neumann (zero flux) boundary conditions are specified. In the $x$ direction, the computed bound states limit to the one-dimensional transverse ground state with the possibility of a phase jump from $-\infty$ to $\infty$. We call the bound states with Dirichlet boundary conditions confined dark solitons (CDSs). Unlike line dark solitons on the unbounded domain $\mathbb{R}^{2}$, or those satisfying Neumann boundary conditions, for the case of impenetrable walls there are no known CDS solutions in analytical form. The CDSs are computed using a spectrally accurate quasi-Newton fixed-point iterative scheme. To accurately analyze the onset of the instability, the NLS/GP equation is linearized around the CDS. The eigenvalues of the linearized equation are found to bifurcate from the origin at a critical confinement width. In particular, when the domain is sufficiently narrow, all eigenvalues are purely imaginary (stable). As the confinement width increases, two purely imaginary eigenvalues coalesce at the origin and emerge as two real (unstable) eigenvalues of opposite signs, whose corresponding 
eigenvectors are antisymmetric along the transverse direction. Dynamically, these eigenvectors induce a transverse "shear" that breaks the bound state apart during propagation. This phenomenon, known as a "snaking" instability, has been shown to give rise to single and multi-vortex excitations $[3,16]$. The critical confinement width obtained at the bifurcation of the discrete spectrum is computed and characterized as a function of the soliton's propagation speed and the type of boundary conditions. We also observe a new bound state solution branch bifurcating from the CDS branch precisely at the critical confinement width. These solutions correspond to single propagating vortices. Using direct numerical simulations of the $(2+1)$-dimensional NLS/GP equation, we show that the critical confinement width coincides with the onset of the transverse instability regime of CDSs. We also show that the $n$-lobed CDSs, $n>1$, exist only for sufficiently wide confinement. These $n$ lobed CDSs are always transversely unstable, leading to the generation of vortices.

We remark that a background flow is present naturally in a shock wave. In particular, dark solitons are intimately related to dispersive shock waves (DSWs), also referred to as collisionless shock waves, dissipationless shock waves, and undular bores [19]. A DSW connects two regions of a flow that possess different parameters, such as density and velocity. Unlike viscous (dissipative) shock waves, a DSW consists of a modulated wavetrain of oscillations. In their seminal work, Gurevich and Pitaevskii developed an asymptotic theory for DSWs, which they used to show that the largest amplitude oscillation of a DSW can be well approximated by a soliton [20]. This work was later extended to the NLS/GP equation where an approximate dark soliton coincides with the slowest edge of the DSW [21]. In addition to solitons, DSWs have been observed experimentally in the aforementioned physical systems as well (cf. [19]). Moreover, the multi-dimensional stability of DSWs has been connected to the stability of the soliton edge [22]. Thus, the results of this study shed light on the dynamics of dark solitons and DSWs.

\section{PROBLEM FORMULATION}

We consider the complex field $\psi(\mathbf{x}, t)$ whose dynamics evolve according to the defocusing (repulsive) NLS/GP equation. In dimensionless form this is

$$
i \psi_{t}+\frac{1}{2} \Delta \psi-|\psi|^{2} \psi=0, \quad \Delta \doteq \partial_{x x}^{2}+\partial_{y y}^{2}
$$

This equation governs the mean field dynamics of a BEC wavefunction, the electric field dynamics of intense laser beams in optical Kerr media, and other nonlinear wave systems. Here, $t>0$ is time, $\mathbf{x}=(x, y)$, where $x \in \mathbb{R}$ is the background flow direction, and $y \in\left(-L_{y} / 2, L_{y} / 2\right)$, which corresponds to an infinitely long $2 \mathrm{D}$ channel of width $L_{y}$. In what follows, we are interested in two kinds of boundary conditions along the transverse direction.

1. Zero-flux walls (Neumann boundary conditions)

$$
\psi_{y}\left(x, \pm L_{y} / 2, t\right)=0 \text {. }
$$

2. Impenetrable walls (Dirichlet boundary conditions)

$$
\psi\left(x, \pm L_{y} / 2, t\right)=0
$$

\section{A. Invariant quantities}

It is expedient to recap some of the well known invariances of (1).

- Galilean invariance with velocity $v \in R$,

$$
\psi^{\prime}\left(x^{\prime}, y, t\right)=\psi\left(x^{\prime}-v t, y, t\right) e^{i\left(v x^{\prime}-v^{2} t / 2\right)} .
$$

- Dilation invariance with amplitude $a \in R$,

$$
\psi^{\prime}\left(x^{\prime}, y^{\prime}, t^{\prime}\right)=a \psi\left(a x^{\prime}, a y^{\prime}, a^{2} t^{\prime}\right)
$$

- Phase invariance with angle $\theta \in[0,2 \pi)$,

$$
\psi^{\prime}\left(x^{\prime}, y^{\prime}, t^{\prime}\right)=e^{i \theta} \psi\left(x^{\prime}, y^{\prime}, t^{\prime}\right)
$$

These invariances are useful for prescribing simplified boundary conditions of the bound state solutions.

As described below, there are conserved quantities that are related to these invariant quantities. However, due to the non-vanishing boundary conditions, the conserved quantities are non-standard. To find them, we first need to define the bound state problem.

\section{B. Bound states and background states}

Given a confinement width $L_{y}$, we seek a oneparameter family of confined dark soliton solutions of (1) in the form

$$
\psi(x, y, t ; \mu)=u_{\mathrm{cds}}(\xi, y ; \mu) e^{-i \mu t}, \quad \xi=x-c t,
$$

where the CDS profile, $u_{\text {cds }}(\cdot)$, is a complex-valued bound state, $c \geq 0$ is the soliton speed, and $\mu$ is the frequency (also called propagation constant or chemical potential [23]). When $c=0$, the confined dark soliton is stationary ("black"). When $c>0$, the confined dark soliton is propagating ("gray"). Inserting the ansatz (6) into (1) gives the nonlinear boundary value problem

$$
\mu u_{\mathrm{cds}}+\frac{1}{2} \Delta u_{\mathrm{cds}}-i c \partial_{x} u_{\mathrm{cds}}-\left|u_{\mathrm{cds}}\right|^{2} u_{\mathrm{cds}}=0,
$$

subject to either of the transverse boundary conditions (2). The boundary conditions for the CDS along the flow direction, i.e., the $x$ axis, are prescribed below. 
Using (3), we may assume, by Galilean invariance (3), that as $x \rightarrow \infty$, the background (far field) flow is stationary. Hence, the speed $c$ plays no role in determining the background flow. Therefore, we require that the bound state approaches a background state as $x \rightarrow \pm \infty$, i.e., the background boundary condition

$$
u_{\mathrm{cds}}(x, y) \stackrel{x \rightarrow \pm \infty}{\longrightarrow} u_{\mathrm{b}, \pm}(y)
$$

where $u_{\mathrm{b}, \pm}(y)$ satisfies the associated ordinary differential equation

$$
\frac{1}{2} u_{\mathrm{b}, \pm}^{\prime \prime}+\mu u_{\mathrm{b}, \pm}-\left|u_{\mathrm{b}, \pm}\right|^{2} u_{\mathrm{b}, \pm}=0
$$

and the same transverse boundary conditions (2) as $u(x, y)$. Here, the background state $u_{\mathrm{b}, \pm}(y)$ is unique up to a complex phase. In fact, the complex phase of $u(x, y)$ varies along the $x$ direction and can approach different values as $x \rightarrow-\infty$ and $x \rightarrow+\infty$. For convenience, we also define

$$
u_{\mathrm{b}}(y) \doteq\left|u_{\mathrm{b}, \pm}(y)\right|
$$

In addition, using (4), we can normalize the density of the background state along the axis of symmetry as

$$
u_{\mathrm{b}}(0)=1
$$

Physically, the background state corresponds to a dispersive (inviscid) generalization of Poiseuille flow for a compressible fluid. We note that the corresponding boundary value problem for the one-dimensional NLS/GP equation was studied in [24].

\section{Particle number}

We consider the initial value problem (1), subject to either of the transverse boundary conditions (2) and the background boundary condition

$$
\psi(x, y, t) \stackrel{x \rightarrow \pm \infty}{\longrightarrow} u_{\mathrm{b}, \pm}(y) e^{-i \mu t}
$$

where $u_{\mathrm{b}, \pm}$ is the background state defined above. We call a solution of this problem a confined dark soliton (CDS). As we shall see, CDS solutions decay rapidly to the background state. Therefore, we define the particle number (in analogy with its interpretation in BECs) as

$$
\mathcal{N}[\psi]=\int_{-\infty}^{\infty} \int_{-L_{y} / 2}^{L_{y} / 2}\left[u_{\mathrm{b}}^{2}(y)-|\psi(x, y, t)|^{2}\right] \mathrm{d} y \mathrm{~d} x
$$

This quantity is finite and positive for CDSs. That this quantity is conserved in time follows from taking its time derivative, using (1), integration by parts, and the boundary conditions.

\section{LINE DARK SOLITON WITH ZERO-FLUX WALLS}

For the case of zero-flux walls (2a), the background is simply constant regardless of the confinement width. It follows from (10), (9), and (7) that $\mu=1$ and $u_{\mathrm{b}}(y) \equiv 1$. Therefore, the exact solution takes the form of a line dark soliton uniform in $y$, i.e.,

$$
\begin{aligned}
\psi(x, y, t ; c) & =\{i c+\nu \tanh [\nu(x-c t)]\} e^{-i t}, \\
\nu^{2}+c^{2} & =1, \quad 0 \leq c<1,
\end{aligned}
$$

up to an overall phase. It is well known that this dark soliton solution exhibits an instability for sufficiently long wavenumber perturbations in the transverse direction [15]. It follows from the critical transverse wavenumber (see [15]) that, in this case, the solution with zero-flux walls is transversely unstable when

$$
L_{y}>L_{\mathrm{cr}}(c)=\frac{\pi}{\sqrt{-1-c^{2}+2 \sqrt{1-c^{2}+c^{4}}}} .
$$

It follows that for dark solitons with zero-flux walls, as the speed increases from 0 to $1, L_{\mathrm{cr}}(c)$ increases monotonically from $\pi$ to $\infty$. Loosely speaking, this means that, as the speed increases, the smaller amplitude line dark soliton becomes more stable to transverse perturbations. This feature has been observed for transverse harmonic potential confinement in $(3+1)$-dimensions [18] and we will see similar behavior for the impenetrable wall boundary condition in what follows.

\section{CDS WITH IMPENETRABLE WALLS}

In many physical systems, transverse boundary conditions can be well approximated by impenetrable walls (Dirichlet boundary conditions) (2b). In this case, there is no exact analytic CDS solution and there is no known exact conditions for stability analogous to (14). However, these CDS solutions can be computed. To do so, it is helpful to obtain approximate analytical solutions as follows. This is achieved below in two steps: first we find the background states and then use them to construct approximate CDSs by an approximate "nonlinear separation of variables", a technique well-known in the BEC community [3].

There is a one-parameter family of solutions of the background state (9) with boundary conditions ( $2 \mathrm{~b}$ ) that can be expressed in terms of a Jacobi elliptic function as

$$
\begin{aligned}
u_{\mathrm{b}}\left(y ; L_{y}\right) & =\operatorname{sn}\left[\kappa\left(y+L_{y} / 2\right), m\right], \\
\kappa & =\frac{1}{\sqrt{m}}, \quad \mu=\frac{1+m}{2 m} .
\end{aligned}
$$

The elliptic parameter $0<m\left(L_{y}\right)<1$ is determined from the boundary conditions according to

$$
L_{y}=2 \sqrt{m} K(m)
$$



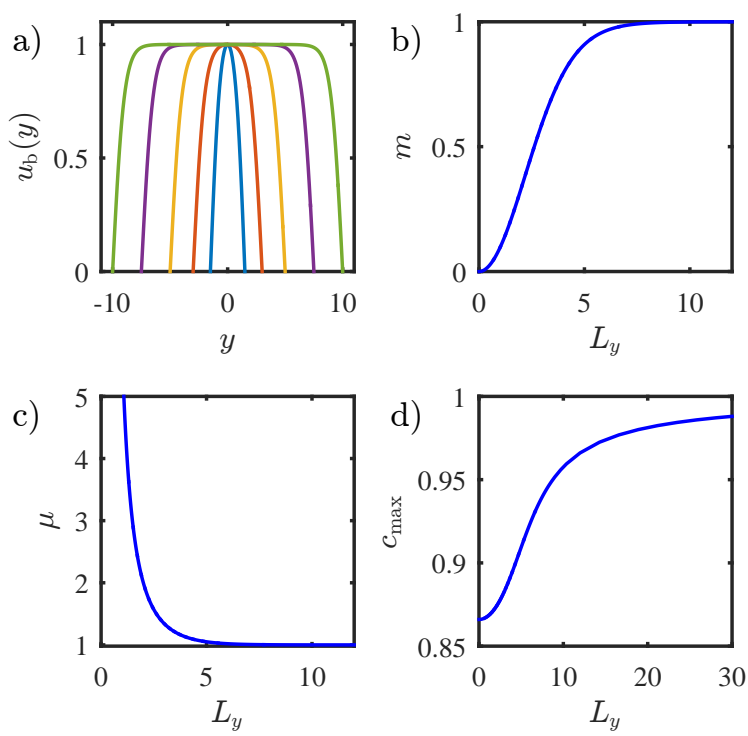

FIG. 1. (Color online) Normalized background states [Eq. (15)] and their salient features. a) Multiple transverse profiles for different widths $L_{y}$. b) Elliptic parameter $m$ [Eq. (16)]. c) Frequency $\mu$ [Eq. (15)]. d) Maximum approximate CDS speed [Eq. (25)].

where $K(m)$ is the complete elliptic integral of the first kind. Thus, given $L_{y}$, we compute $m$ using (16) and find $\mu, \kappa$, and $u_{\mathrm{b}}$ using (15). Figure III depicts several such background states as well as the elliptic parameter, frequency, and maximal velocity (see below) for varying confinement widths. In particular, Fig. III(c) shows that as the confinement width increases, the frequency converges rapidly to its unconfined value, which is the same as that of Neumann (constant flux) transverse boundary conditions, i.e., $\lim _{L_{y} \rightarrow \infty} \mu=1$. Note that in this limit, formally, $m \rightarrow 1$ and $\operatorname{sn}(\cdot ; m) \rightarrow \tanh (\cdot)$. However, this is a singular limit and, in fact, the limiting $u_{\mathrm{b}}$ is constant along the $y$ direction.

In addition, it can be shown from (15) that

$$
\int_{-L_{y} / 2}^{L_{y} / 2} u_{\mathrm{b}}^{2}(y) \mathrm{d} y=\frac{2}{\sqrt{m}}[K(m)-E(m)],
$$

where $E(m)$ is the complete elliptic integral of the second kind. These relations are useful for the subsequent analysis and computations.

\section{A. Approximate analytical CDS with impenetrable walls}

Because the NLS/GP equation is nonlinear, strictly speaking, linear methods for partial differential equations, such as the method of separation of variables, do not apply for this equation. In spite of this, it is shown below that one can obtain an approximate separable solution, which, in turn, is found to be fairly accurate and useful. Such an approach has been shown to work well for a variety of transverse configurations, including tight harmonic confinement [3]. Intuitively, for sufficiently tight confinement, the energy to excite transverse dynamics is large and one expects the transverse direction to be "frozen".

Thus, we seek a solution of (1) and (2b) in the separable form

$$
\psi\left(x, y, t ; c, L_{y}\right)=u_{\mathrm{b}}\left(y ; L_{y}\right) \tilde{\psi}(x, t ; c)
$$

where $u_{\mathrm{b}}$ is the background solution (15). Inserting this ansatz into (1), multiplying by $u_{\mathrm{b}}$, and integrating over $y$, yields

$$
i \frac{\partial \tilde{\psi}}{\partial t}+\frac{1}{2} \frac{\partial^{2} \tilde{\psi}}{\partial x^{2}}-\alpha|\tilde{\psi}|^{2} \tilde{\psi}=0
$$

where the nonlinear coefficient is determined through the confinement width $L_{y}$ as

$$
\begin{aligned}
\alpha\left(L_{y}\right) & =\frac{\int_{0}^{K(m)} \operatorname{sn}^{4}(y, m) d y}{\int_{0}^{K(m)} \operatorname{sn}^{2}(y, m) d y} \\
& =\frac{1}{3}\left[1+\frac{2}{m}+\frac{E(m)}{E(m)-K(m)}\right] .
\end{aligned}
$$

It follows from (20) that

$$
\lim _{L_{y} \rightarrow 0} \alpha=\lim _{m \rightarrow 0} \alpha=\frac{3}{4} .
$$

The physical meaning of $\alpha$ is an effective nonlinear coupling constant (or scattering length in BECs). In the unconfined case, $\nu^{2}+c^{2}=\alpha^{2}=1$. In the confined case, $\alpha<1$, i.e., there is a reduced speed that a dark soliton can have due to the confinement, which has been observed experimentally in BECs [12].

Equation (19) admits dark soliton solutions that we use in order to construct a family of approximate CDS solutions propagating with speed $c$ as

$$
\begin{gathered}
\psi_{\text {approx. }}(x, y, t)=u_{\mathrm{b}}(y) \frac{i c+\nu \tanh [\nu \xi]}{\sqrt{\alpha}} e^{-i \mu t}, \\
\nu=\sqrt{\alpha-c^{2}}, \quad \mu=\alpha, \quad 0 \leq c<\sqrt{\alpha} .
\end{gathered}
$$

We note that the approximate CDS (22) does not satisfy the NLS/GP equation exactly, though it does satisfy the boundary conditions (2b), (8), and (10). The far-field behavior

$$
\lim _{x \rightarrow \pm \infty} \psi_{\text {approx. }}(x, 0, t)=\frac{i c \pm \nu}{\sqrt{\alpha}} e^{-i \mu t},
$$

implies the phase jump

$$
\Delta \phi=\pi-2 \tan ^{-1}\left(c / \sqrt{\alpha-c^{2}}\right)
$$


as the dark soliton is traversed. Furthermore, it follows from (22) that for a given confinement width, $L_{y}$, there is a maximal speed given by

$$
c_{\max }\left(L_{y}\right)=\sqrt{\alpha\left(L_{y}\right)} .
$$

Figure III(c) shows the variation of this maximal speed with the confinement width. As $c \rightarrow c_{\max }\left(L_{y}\right)$, the CDS (22) approaches the uniform in- $x$ background state, $u_{\mathrm{b}}(y)$. It follows from (21) that

$$
\sqrt{3} / 2 \leq c_{\max }<1 .
$$

We also find the particle number (12) for the approximate CDS as

$$
\mathcal{N}\left[\psi_{\text {approx. }}\right]=\frac{4}{\alpha \sqrt{m}}[K(m)-E(m)] \sqrt{\alpha-c^{2}} .
$$

We remark that there are other approaches in the literature to obtain approximate solutions, e.g., the Lagrangian approach [3]. In all cases, the approximation cannot satisfy exactly both the NLS/GP equation and the boundary conditions. The method above was chosen because it satisfies the boundary conditions exactly. The computations discussed below verify the utility of this approach.

\section{B. CDS computations}

The CDS solutions with impenetrable walls are computed using the spectrally accurate quasi-Newton iterative method described in Appendix A. The approximate analytic CDS described above is used as an initial guess for these iterations. Simple continuation of the computed CDS solutions is used for larger $L_{y}$. Figure 2 presents a comparison of cross-sections between an approximate CDS and the numerically computed one, demonstrating good agreement for sufficiently tight confinement. This is further confirmed in Fig. 3a where the error between the approximate and numerical CDS densities for a fixed speed is shown to decrease with decreasing $L_{y}$. Figure $3 \mathrm{~b}$ gives the error for fixed width and variable speed. In addition, Fig. 4 shows that the phase jump across the approximate CDS is fairly accurate compared to the numerically computed CDS for a wide range of confinement widths and propagation speeds. This underscores the utility of the approximate CDS.

In addition, CDS solutions can possess any number of oscillation lobes within the central depression. Figure 5 presents examples of gray (propagating) solitons with speed $c=0.5$ for varying confinement widths, which possess one, two, and three lobes. The maximal possible number of lobes depends on $c$ and $L_{y}$. The entire family of 1-lobe CDSs can be computed using a continuation method in $L_{y}$ - these CDSs bifurcate from the uniform background state $u_{\mathrm{b}}(y)$. For $n>1$, we find that an $n$-lobed CDS exists if the confinement width $L_{y}$ is
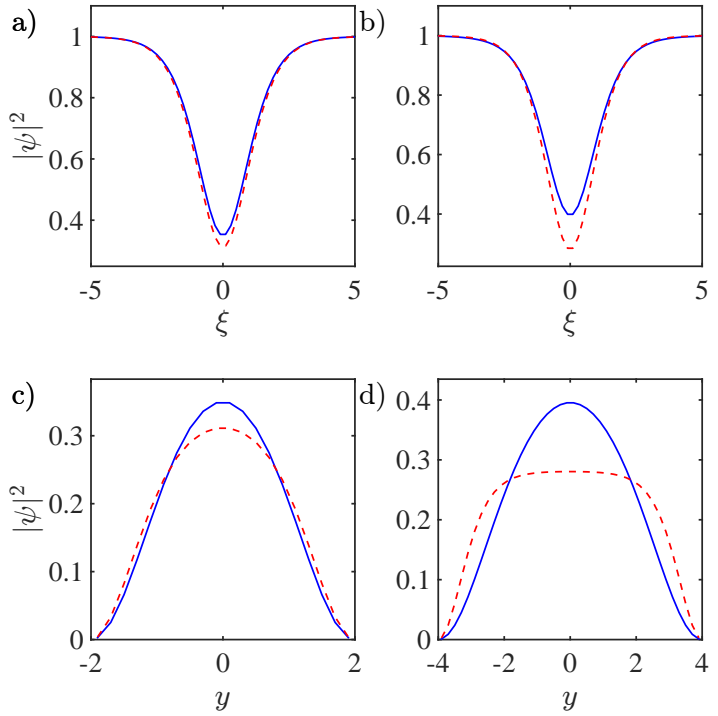

FIG. 2. (Color online) Comparison of approximate (dashed) and exact (solid) CDS cross sections for $c=0.5$. (a,b) Longitudinal cross sections along $y=0$ with transverse confinement widths $L_{y}=4$ and $L_{y}=8$, respectively. (c,d) Transverse cross sections along $\xi=0$ with transverse confinement widths $L_{y}=4$ and $L_{y}=8$, respectively. a)

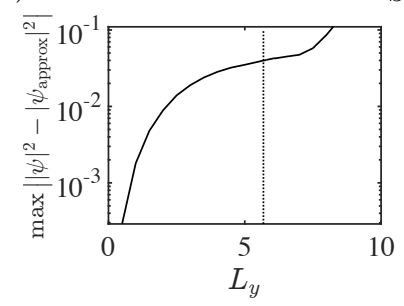

b)

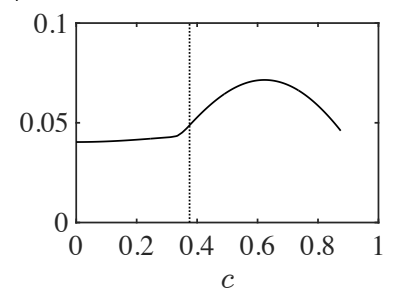

FIG. 3. Maximum absolute error in density $|\psi|^{2}$ between approximate and numerical CDSs. a) Fixed $c=0.25$, variable width $L_{y}$. b) Fixed $L_{y}=6$, variable speed $c$. The dotted vertical line separates unstable and stable CDS solutions (see Sec. IV).

above a critical value, $L_{\mathrm{th}}^{n}(c)$. When the Newton solver is initialized with the approximate (1-lobe) CDS (22) for $L_{\mathrm{th}}^{n}(c)<L_{y}<L_{\mathrm{th}}^{n+1}(c)$, the iteration converges to an $n$-lobe CDS. Continuation along each $n$-lobe branch enables the numerical determination of $L_{\mathrm{th}}^{n}(c)$. For example, $L_{\mathrm{th}}^{2}(0.5) \approx 12.8$ and $L_{\mathrm{th}}^{3}(0.5) \approx 21.8$. We interpret these lobed solutions as "nonlinear excited states" of the channel.

\section{Bifurcation of CDS families and vortex solitons}

To better understand the properties of the different CDS families, Fig. 6 depicts the particle number $\mathcal{N}$ for 
a)

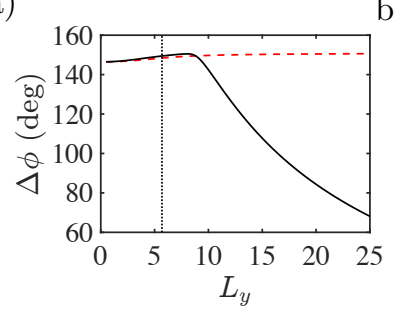

b)

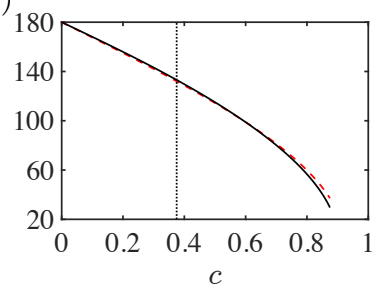

FIG. 4. (Color online) Dependence of the phase jump across the CDS solution as a) the confinement width $L_{y}$ is varied with fixed CDS speed $c=0.25$ and b) as the speed $c$ is varied with fixed width $L_{y}=6$. These plots show deviation from the approximate CDS phase jump (dashed) for large $L_{y}$. The dotted vertical line separates unstable and stable CDS solutions (see Sec. IV).
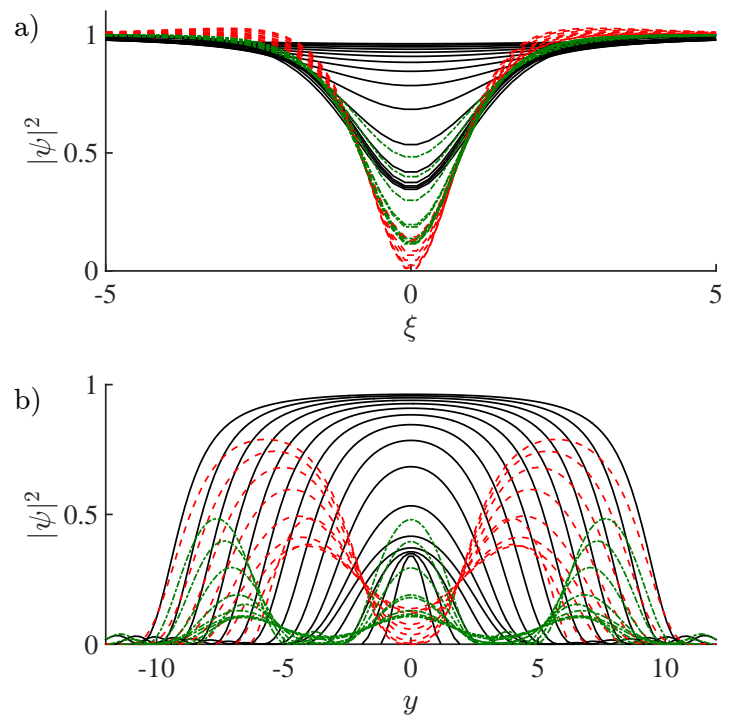

FIG. 5. (Color online) Cross sections along a) the longitudinal direction for $y=0$ and $\mathrm{b}$ ) along the transverse direction with $\xi=0$ of single-lobed (solid black), double-lobed (dashed red), and triple-lobed (dash-dotted green) CDSs with propagation speed $c=0.5$ and a range of confinement widths $L_{y}$.

the 1-, 2-, and 3-lobed CDSs as well as for the vortex. The 1-lobed CDSs bifurcate from the uniform background state for which $\mathcal{N}\left[u_{\mathrm{b}}\right]=0$, whereas, the $n$-lobed CDS bifurcates from a suitably close approximate CDS (dashed red curve) with a sufficiently large particle number.

In addition, our CDS computations reveal solitonic vortices. Vortices have been known and studied extensively for many years. In most cases, vortices arise in pairs of opposite circulation such that their total angular momentum vanishes. However, single solitonic vortices have been predicted and studied theoretically (cf. [16, 25$32]$ ). Our study here focuses on two-dimensional vortices that have been studied extensively in BECs (see, e.g., [3] and references therein). We note that the

three-dimensional counterpart, a vortex line, has been fairly elusive yet has been observed recently in Fermi gases [33, 34] and BECs [35]. In addition to the aforementioned lobed CDSs, Fig. 6 presents the particle number for solitonic vortex solutions with varying confinement widths. We note that the particle number of solitonic vortices is equal or lower than for the 1-lobed CDS. This suggests that solitonic vortices should be persistent structures, even for wide channels.

In addition, Fig. 7 presents the particle numbers for 1lobe CDSs and vortex solitons with varying propagation speeds. In this figure, the stable and unstable modes are distinguished by different curve types. We now describe the stability properties of these solutions.

\section{STABILITY ANALYSIS AND DIRECT NUMERICAL SIMULATIONS}

As mentioned in Section II, the critical confinement width for transverse stability of line dark solitons with zero-flux walls (Neumann boundary conditions) is given analytically by eq. (14). However, there is no such simple expression for CDS with impenetrable walls (Dirichlet boundary conditions). Here we investigate the linear (spectral) transverse stability of the CDSs.

In Appendix B the linearized NLS/GP equation around a CDS solution is derived (also termed the Bogoliubov-de Gennes equations in BEC studies). We compute the spectrum of the linearized equation for varying confinement width $L_{y}$. For a given soliton speed, $c$, when the domain is sufficiently narrow, the spectrum of the linearized operator consists of purely imaginary (stable) discretized eigenvalues and continuous spectrum (radiation modes). When the confinement width reaches a critical value, $L_{y}=L_{\mathrm{cr}}(c)$, two purely imaginary eigenvalues coalesce at the origin. For $L_{y}>L_{\mathrm{cr}}(c)$, these eigenvalues emerge as two real (unstable) eigenvalues of opposite signs, whose corresponding eigenvectors are antisymmetric along the transverse direction. Figure 8[(a) and (b)] depicts this bifurcation when $c=0.5$ for which $L_{\text {cr }} \approx 6.55$. Figure $8(\mathrm{c})$ shows the intensity and complex phase of one of the unstable eigenfunctions. Whereas the intensity is symmetric, the complex phase is odd in both $x$ and $y$, which gives rise to the "snaking" instability observed in the dynamics.

Our computations of the linearized spectrum reveal that the 1-lobed CDSs are stable when they are sufficiently confined, i.e., for $L_{y}<L_{\mathrm{cr}}(c)$, where $L_{\mathrm{cr}}(c)$ is the critical confinement width. Note that the background solution $u_{b}(y)$ is linearly stable. Figure ?? presents a plot of the critical confinement width $L_{\mathrm{cr}}(c)$ for both Neumann and Dirichlet boundary conditions. In general, we find that

$$
L_{\mathrm{cr}}(c) \geq L_{\mathrm{cr}}(0) \approx 5.5, \quad 0 \leq c<1 .
$$

The result by [16] for black solitons was that $L_{\mathrm{cr}}(0) \approx$ 6 , which is close to the value we obtain using spectral 

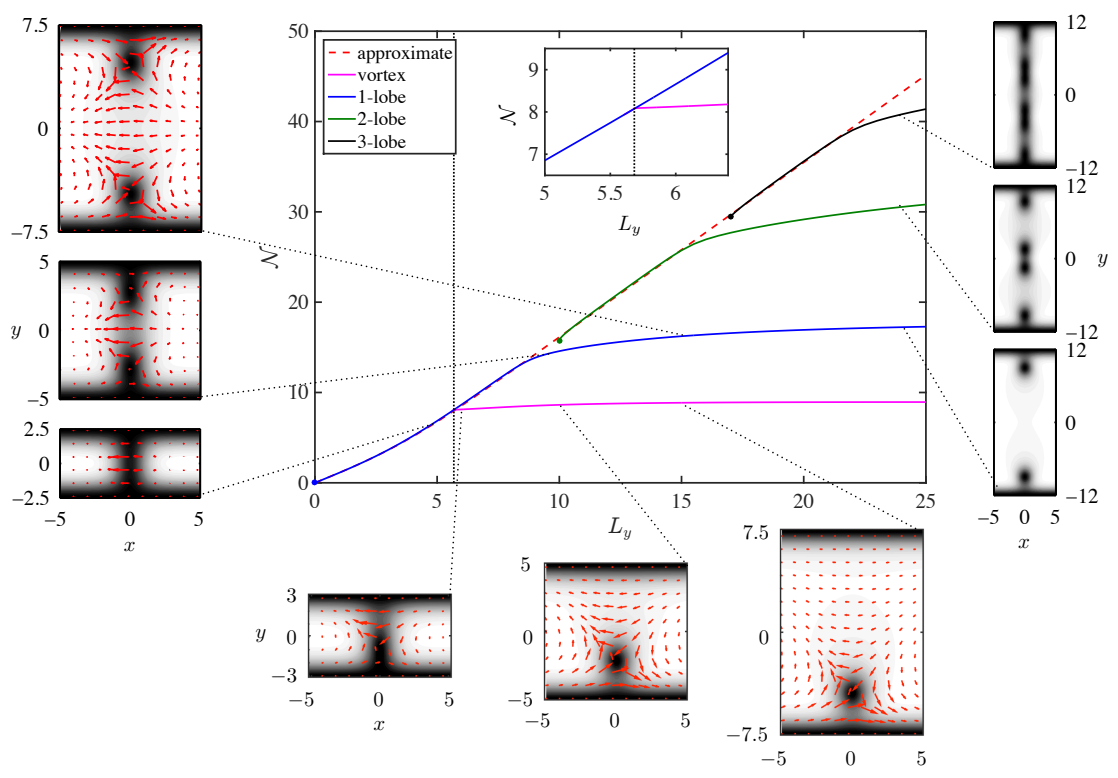

FIG. 6. (Color online) Phase diagram depicting the conserved quantity $\mathcal{N}$ and contour plots of 1, 2, and 3-lobed channel CDS and solitonic vortex solutions with fixed $c=0.25$ as $L_{y}$ is varied. The dotted vertical line corresponds to the critical confinement width $L_{\mathrm{cr}} \approx 5.68$ below which CDS solutions are stable (see Sec. IV). The critical width corresponds to the minimal $L_{y}$ such that the solitonic vortex exists.

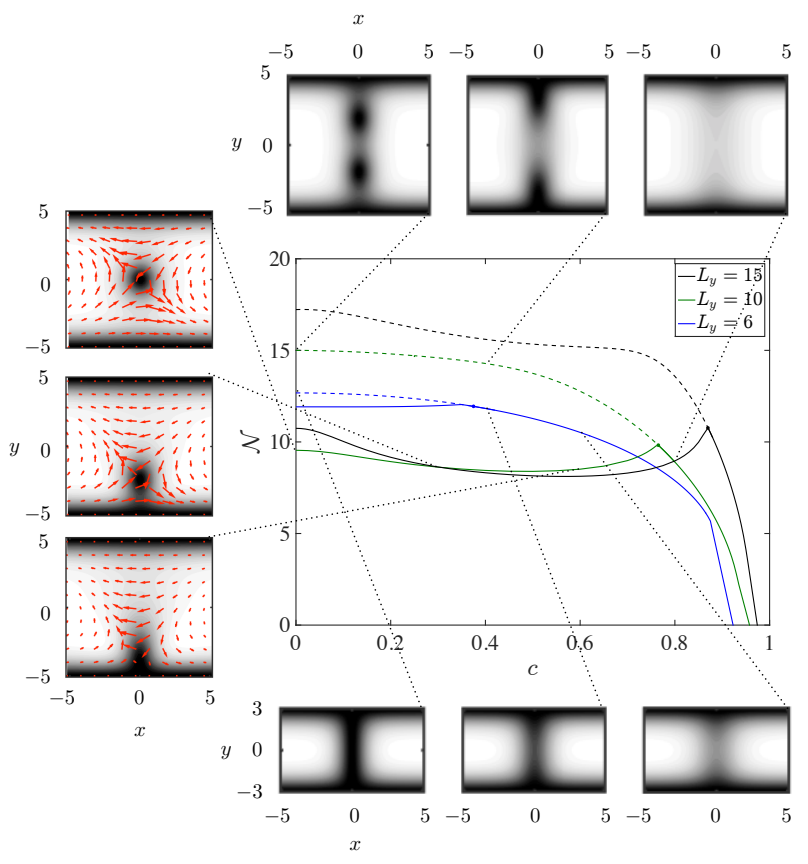

FIG. 7. (Color online) Phase diagram depicting the conserved quantity $\mathcal{N}$ and contour plots of 1-lobed CDS and vortex solutions with variable speeds $c$ and several confinement widths $L_{y}$. Filled circles correspond to the crossover from unstable (dashed curves) to stable (solid curves) CDS solutions (see Sec. IV).

analysis.

The inset of Fig. 6 shows that the solitonic vortex so-
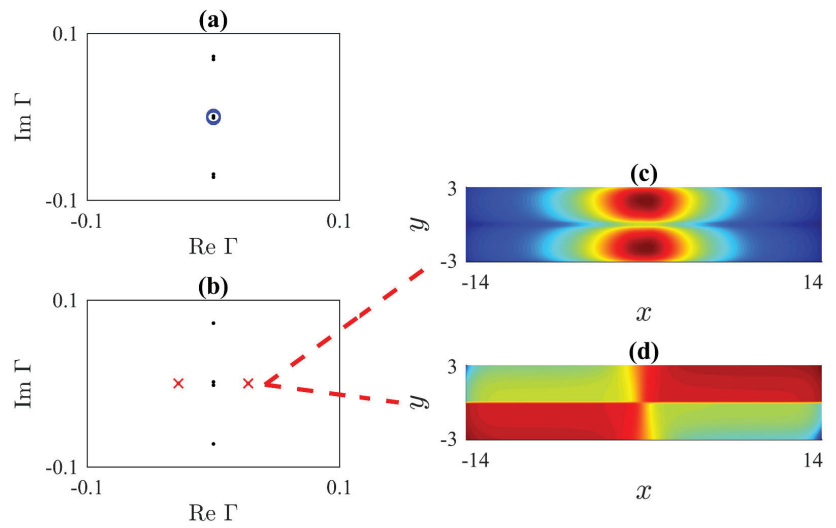

(d)

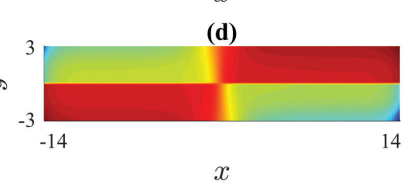

FIG. 8. (Color online) A portion around the complex origin of the linearized spectra of a CDS with speed $c=0.5$ and confinement widths (a) $L_{y}=6.4$ and (b) $L_{y}=6.6$. Two stable (purely imaginary) eigenvalues [denoted by $\odot$ in (a) and almost indistinguishable] coalesce at the origin and emerge as unstable (real) eigenvalues [denoted by $\times$ in (b)]. Contour plots of the intensity (c) and phase (d) of one of the unstable eigenfunctions.

lution branch bifurcates from the 1-lobe CDS solution branch precisely at $L_{\mathrm{cr}}$. We can therefore interpret the onset of the 1-lobe CDS instability as precisely the confinement width at which a lower particle number solitonic vortex solution appears. This interpretation can also be corroborated by dynamical evolution of the 1-lobe CDS described below.

In Fig. 7 above, the stable and unstable modes were 


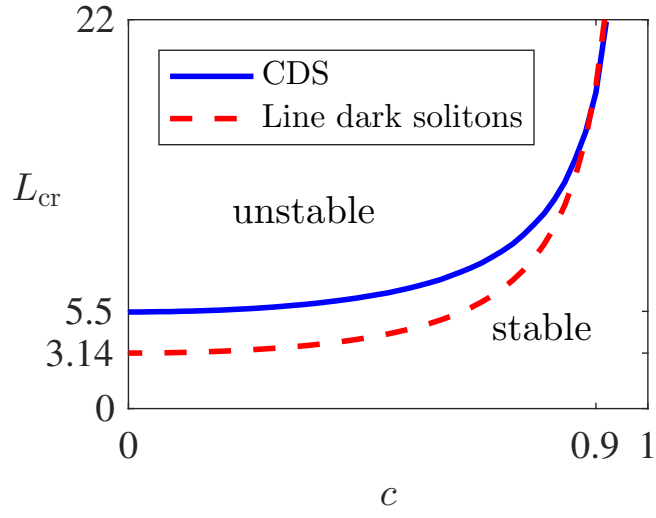

FIG. 9. (Color online) Critical confinement widths as functions of propagation speed, $L_{\mathrm{cr}}(c)$, for line dark solitons with constant-flux walls (dashed) and CDSs with impenetrable walls (solid). For $L_{y}>L_{\mathrm{cr}}(c)$ the solutions are unstable.

distinguished by different curve types. For a given confinement width, the modes become unstable when their particles number increases beyond a critical threshold. In general, the multi-lobed CDSs are all unstable.

Finally, to test the linear stability theory, we carry out direct numerical simulations of the NLS/GP equation 1 with impenetrable walls and initial conditions that correspond to a CDS with a small amount of "noise". The numerical method is explained in Appendix C. Figure 10 presents some of the results, which show that $L_{\mathrm{cr}}(c)$, obtained from spectral analysis, is indeed the critical confinement width for nonlinear stability. Moreover, these simulations reveal that, when $L_{y}>L_{\mathrm{cr}}$, the CDS undergoes a snaking instability and can break up into a single solitonic vortex as in Fig. (b) or counterpropagating solitonic vortex pairs as in Fig. (c).

\section{CONCLUSIONS}

The critical confinement widths for stabilizing propagating dark solitons in the defocusing/repulsive NLS/GP equation were obtained using spectral analysis and verified by direct computations. The results show that: (i) for a given confinement width, the faster the solitons propagate, the more stable they become; (ii) impenetrable walls are more stabilizing than zero flux boundaries. These results generalize upon previous studies for black (non-propagating) dark solitons and gray (propagating) dark solitons. As part of this analysis, we also analytically obtained approximate confined dark solitons with impenetrable walls. This approximation was used to show that confined dark solitons have a reduced speed compared with the unconfined case, which is consistent with and may help to explain experimental observations in BECs.

\section{COMPUTATIONAL METHODS}

\section{Appendix A: Computing the CDS}

We compute CDS bound state solutions of (7) and (2b) using a spectrally accurate quasi-Newton approach [36]. It is convenient to break up (7) into its real and imaginary parts by taking $u_{\mathrm{cds}}=u+i v$ where $u, v$ are real valued

$$
\begin{aligned}
& F(u, v)=\frac{1}{2} \Delta u+c v_{\xi}-\left(u^{2}+v^{2}-\mu\right) u=0, \\
& G(u, v)=\frac{1}{2} \Delta v-c u_{\xi}-\left(u^{2}+v^{2}-\mu\right) v=0 .
\end{aligned}
$$

We seek solutions that rapidly asymptote to the uniform background state

$$
u^{2}(\xi, y)+v^{2}(\xi, y) \rightarrow u_{\mathrm{b}}(y)^{2}, \quad|\xi| \rightarrow \infty
$$

The far field density normalization (10) determines the chemical potential $\mu$ to be its background value (A4).

We solve eq. (A1) using discrete cosine/sine transforms achieving rapid (spectral) convergence. For completeness, we include a discussion of these transforms in the following subsection.

\section{Discrete transforms}

In order to evaluate eq. (7) on a finite grid with spectral accuracy, we introduce the half grid points on $\left[-L_{\xi, y} / 2, L_{\xi, y} / 2\right]$

$$
\begin{aligned}
& \xi_{i}=-\frac{L_{\xi}}{2}+\frac{2 i-1}{2} \Delta_{\xi}, \quad \Delta_{\xi}=\frac{L_{\xi}}{N_{\xi}}, \quad i=1,2, \ldots, N_{\xi}, \\
& y_{j}=-\frac{L_{y}}{2}+\frac{2 j-1}{2} \Delta_{y}, \quad \Delta_{y}=\frac{L_{y}}{N_{y}}, \quad j=1,2, \ldots, N_{y} .
\end{aligned}
$$

The truncation of $\mathbb{R}$ to $\xi \in\left(-L_{\xi} / 2, L_{\xi} / 2\right)$ is achieved by employing Neumann boundary conditions

$$
u_{\xi}\left( \pm L_{\xi} / 2, y\right)=0, \quad v_{\xi}\left( \pm L_{\xi} / 2, y\right)=0
$$

which are natural for dark solitons that rapidly decay to differing constant values for $x \rightarrow \pm \infty$. To evaluate derivatives and simultaneously satisfy the boundary conditions, we approximate the solution with a truncated cosine series expansion in $\xi$ and a truncated sine series 
a)

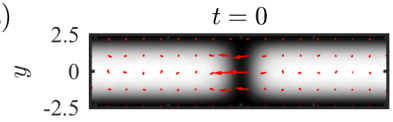

b)

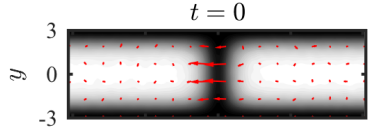

c)

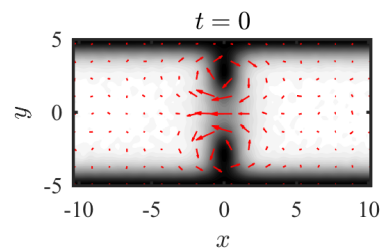

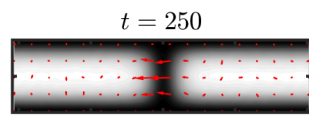

$t=250$
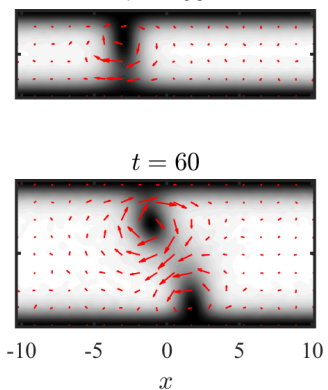

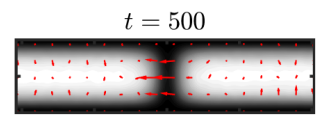

$t=500$
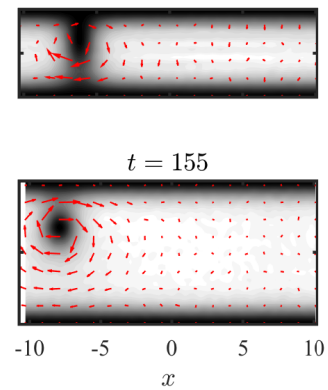

FIG. 10. Stable and unstable dynamics for initially perturbed 1-lobed CDS solutions of speed $c=0.25$ evolved according to the NLS equation (1). a) Stable dynamics for $L_{y}=5$. b) Unstable dynamics leading to a single vortex for $L_{y}=6$. c) 1 -lobe CDS decay into two vortices resulting from the wider channel $L_{y}=10$. The mean zero noise variance is $\sigma=10^{-4}$. Note that $L_{\mathrm{cr}}(0.25) \approx 5.68$ so the instability transition is accurately resolved.

expansion in $y$

$$
\begin{array}{r}
u(\xi, y) \approx \\
\frac{2}{\left(N_{\xi} N_{y}\right)^{1 / 2}} \sum_{n=1}^{N_{x}} \sum_{m=1}^{N_{y}} \hat{u}_{n, m} \cos \left(\frac{\pi\left(\xi+L_{\xi} / 2\right)(n-1)}{L_{\xi}}\right) \\
\times \sin \left(\frac{\pi\left(y+L_{y} / 2\right)(m-1)}{L_{y}}\right),
\end{array}
$$

$v(\xi, y) \approx$

$$
\begin{array}{r}
\frac{2}{\left(N_{\xi} N_{y}\right)^{1 / 2}} \sum_{n=1}^{N_{x}} \sum_{m=1}^{N_{y}} \hat{v}_{n, m} \\
\quad \cos \left(\frac{\pi\left(\xi+L_{\xi} / 2\right)(n-1)}{L_{\xi}}\right) \\
\times \sin \left(\frac{\pi\left(y+L_{y} / 2\right)(m-1)}{L_{y}}\right) .
\end{array}
$$

The prime on the first summation implies that whenever $n=1$, the coefficient should be divided by $\sqrt{2}$. The Fourier coefficients are computed using fast cosine and sine transforms (DCT-II and DST-II) [37] from the discrete function values at the half grid points. The fast transforms are implemented by appropriate pre and post processing of the FFT.
Derivatives are approximated according to

$$
\begin{aligned}
u_{\xi} & \approx \mathcal{S}_{k_{\xi}}^{-1}\left\{-k_{\xi} \mathcal{C}_{\xi}\left\{u_{\mathrm{cds}}\right\}\right\}, \\
u_{\xi \xi} & \approx \mathcal{C}_{k_{\xi}}^{-1}\left\{-k_{\xi}^{2} \mathcal{C}_{\xi}\left\{u_{\mathrm{cds}}\right\}\right\}, \\
u_{y y} & \approx \mathcal{S}_{k_{y}}^{-1}\left\{-k_{y}^{2} \mathcal{S}_{y}\left\{u_{\mathrm{cds}}\right\}\right\},
\end{aligned}
$$

where the notation $\mathcal{C}_{\xi}$ refers to a DCT in $\xi$ and $\mathcal{S}_{k_{\xi}}^{-1}$ refers to an inverse DST in $k_{\xi}$, etc. The discrete wavenumbers are

$$
k_{\xi, y} \in \frac{\pi}{L_{\xi, y}}\left\{0,1, \ldots, N_{\xi, y}\right\} .
$$

We use a black-box Newton solver on a preconditioned version of eq. (A1), seeking $u, v$ such that $L^{-1} \mathbf{F}(u, v)=$ 0 . The preconditioner we use is

$$
\begin{aligned}
& L=\left[\begin{array}{cc}
a-\mu-\frac{1}{2} \Delta & -c \partial_{\xi} \\
c \partial_{\xi} & a-\mu-\frac{1}{2} \Delta
\end{array}\right], \\
& L^{-1}=\left[\left(a-\mu-\frac{1}{2} \Delta\right)^{2}+c^{2} \partial_{\xi \xi}\right]^{-1} \\
& \times\left[\begin{array}{cc}
a-\mu-\frac{1}{2} \Delta & -c \partial_{\xi} \\
c \partial_{\xi} & a-\mu-\frac{1}{2} \Delta
\end{array}\right],
\end{aligned}
$$

where $a>0$ is an acceleration parameter. The preconditioner is applied efficiently using the DCST

$$
L^{-1} \mathbf{F}=\left[\begin{array}{l}
\mathcal{S}_{k_{y}}^{-1} \mathcal{C}_{k_{\xi}}^{-1}\left\{\frac{a-\mu+\frac{1}{2}\left(k_{\xi}^{2}+k_{y}^{2}\right)}{\left(a-\mu+\frac{1}{2}\left(k_{\xi}^{2}+k_{y}^{2}\right)\right)^{2}-c^{2} k_{\xi}^{2}} \mathcal{S}_{y} \mathcal{C}_{\xi}\{F\}\right\}+\mathcal{S}_{k_{y}}^{-1} \mathcal{S}_{k_{\xi}}^{-1}\left\{\frac{-c k_{\xi}}{\left(a-\mu+\frac{1}{2}\left(k_{\xi}^{2}+k_{y}^{2}\right)\right)^{2}-c^{2} k_{\xi}^{2}} \mathcal{S}_{y} \mathcal{C}_{\xi}\{G\}\right\} \\
\left.\mathcal{S}_{k_{y}}^{-1} \mathcal{C}_{k_{\xi}}^{-1}\left\{\frac{a-\mu+\frac{1}{2}\left(k_{\xi}^{2}+k_{y}^{2}\right)}{\left(a-\mu+\frac{1}{2}\left(k_{\xi}^{2}+k_{y}^{2}\right)\right)^{2}-c^{2} k_{\xi}^{2}} \mathcal{S}_{y} \mathcal{C}_{\xi}\{G\}\right\}-\mathcal{S}_{k_{y}}^{-1} \mathcal{S}_{k_{\xi}}^{-1}\left\{\frac{-c k_{\xi}}{\left(a-\mu+\frac{1}{2}\left(k_{\xi}^{2}+k_{y}^{2}\right)\right)^{2}-c^{2} k_{\xi}^{2}} \mathcal{S}_{y} \mathcal{C}_{\xi}\{F\}\right\}\right]
\end{array}\right.
$$

The stopping (convergence) criteria for Newton's method is an $l^{2}$ norm of the residual less than $10^{-13}$. 


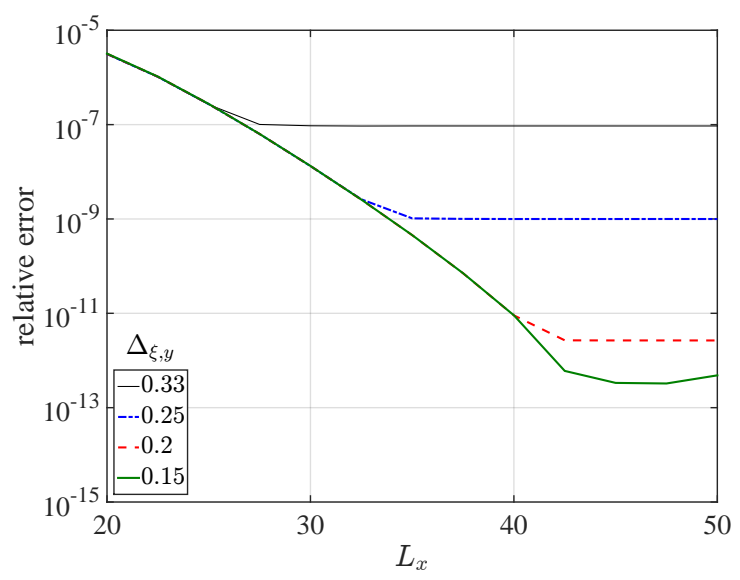

FIG. 11. (Color online) Typical convergence of Newton calculations with $c=0.5, L_{y}=7$ with varying grid spacing $\left(\Delta_{\xi, y}\right.$ in the legend) and longitudinal length $L_{x}$.

An example convergence plot is presented in Fig. 11. The reference "exact" solution is computed on a large $\left(L_{x}=60\right)$, very fine grid $(\Delta=0.1)$. The black soliton $(c=0)$ exhibits the strongest localization and requires $L_{x} \approx 40$ to achieve the highest accuracy. The gray soliton with $c=0.5$ is broader than the dark soliton and hence requires a slightly longer channel width $L_{x} \approx 50$ to achieve the highest accuracy. For both the black and gray solitons a grid spacing of $\Delta x=\Delta y=0.15$ achieves an absolute accuracy of $10^{-13}$. For all calculations in this study, we used $L_{x}=40$ and grid spacing $\Delta=0.2$, ensuring absolute errors below $10^{-10}$.

\section{Appendix B: Linearization}

Given a stationary solution of (1) via eq. (7), its linearization is of interest for Newton solver implementations and for computing spectral stability. Inserting the ansatz

$$
\begin{aligned}
\psi(x, y, t)=[ & u(\xi, y)+\varepsilon f(\xi, y) e^{\Gamma t} \\
& \left.+i\left(v(\xi, y)+\varepsilon g(\xi, y) e^{\Gamma t}\right)\right] e^{-i \mu t}
\end{aligned}
$$

into eq. (1) with $u, v$ a solution of (A1) $F(u, v)=$ $G(u, v)=0$ and keeping only $\mathcal{O}(\varepsilon)$ terms results in the linearization

$$
\Gamma\left[\begin{array}{l}
f \\
g
\end{array}\right]=\left[\begin{array}{cc}
0 & -1 \\
1 & 0
\end{array}\right] \mathcal{J}(u, v)\left[\begin{array}{l}
f \\
g
\end{array}\right],
$$

where $\mathcal{J}=\partial[F, G] / \partial[u, v]$ is the Jacobian of the nonlinear system (7)

$\mathcal{J}(u, v)=\left[\begin{array}{cc}\frac{1}{2} \Delta-\left(3 u^{2}+v^{2}-\mu\right) & c \partial_{\xi}-2 u v \\ -c \partial_{\xi}-2 u v & \frac{1}{2} \Delta-\left(u^{2}+3 v^{2}-\mu\right)\end{array}\right]$.

Note that $\mathcal{J}$ is self-adjoint.

\section{Appendix C: Dynamical evolution}

In this section we present a numerical scheme to efficiently solve the NLS/GP equation (1) for the case of a channel with impenetrable walls (2b). We use a split-step pseudospectral method.

We consider (1) in the reference frame moving with the background flow of velocity $c$ in the $x$ direction

$$
\begin{aligned}
i \psi_{t} & =-\frac{1}{2}\left(\psi_{x x}+\psi_{y y}\right)+i c \psi_{x}+|\psi|^{2} \psi, \\
\psi(x, y, 0) & =\psi_{0}(x, y), \quad \psi\left(x, \pm L_{y} / 2, t\right)=0 .
\end{aligned}
$$

The far field behavior (8) implies non-homogeneous Dirichlet boundary conditions for $\psi$. However, for splitting methods, it is typically advantageous to use homogeneous boundary conditions. To this end, we introduce Neumann boundary conditions in the $x$ direction as

$$
\psi_{x}\left( \pm L_{x} / 2, y, t\right)=0 .
$$

If we assume sufficient localization in the $x$ direction, the condition $(\mathrm{C} 2)$ is consistent with the background state 8 for sufficiently large $L_{x}$.

The standard split-step method is comprised of two steps as follows.

Nonlinear step: $\quad i \psi_{t}=|\psi|^{2} \psi, \quad \psi(x, y, 0)=\psi_{0}(x, y)$

and

$$
\begin{aligned}
& \text { Linear step: } i \psi_{t}=-\frac{1}{2}\left(\psi_{x x}+\psi_{y y}\right)+i c \psi_{x}, \\
& \psi(x, y, 0)=\psi_{0}(x, y), \quad \psi\left(x, \pm L_{y} / 2, t\right)=0 \\
& \psi_{x}\left( \pm L_{x} / 2, y, t\right)=0
\end{aligned}
$$

The nonlinear step is an ODE, which can be solved exactly as

$$
\psi(x, y, t)=e^{-i\left|\psi_{0}(x, y)\right|^{2} t} \psi_{0}(x, y) .
$$

The linear step can be solved with spectral accuracy as

$$
\begin{aligned}
\psi(x, y, t) & =e^{i t \Delta / 2} \psi_{0}(x+c t, y) \\
& =\mathcal{C}_{k_{x}}^{-1} \mathcal{S}_{k_{y}}^{-1}\left\{e^{-i t\left(k_{x}^{2}+k_{y}^{2}\right) / 2} \mathcal{C}_{x} \mathcal{S}_{y}\left\{\psi_{0}(\cdot+c t, \cdot)\right\}\right\} .
\end{aligned}
$$

where trigonometric interpolation is used in order to evaluate $\psi_{0}(x+c t, y)$. The solutions of these two problems are then chained together in order to achieve $2^{\text {nd }}$ order accuracy in time by taking successive half nonlinear, full linear, and half nonlinear steps. 
[1] Y. S. Kivshar and D. E. Pelinovsky, Phys. Rep. 331, 117 (2000).

[2] D. J. Frantzeskakis, J. Phys. A 43, 213001 (2010).

[3] P. G. Kevrekidis, D. J. Frantzeskakis, and R. Carretero-González, The Defocusing Nonlinear Schrödinger Equation (SIAM, 2015).

[4] P. Emplit, J.-P. Hamaide, F. Reynaud, C. Froehly, and A. Barthelemy, Opt. Comm. 62, 374 (1987).

[5] A. M. Weiner, J. P. Heritage, R. J. Hawkins, R. N. Thurston, E. M. Kirschner, D. E. Leaird, and W. J. Tomlinson, Phys. Rev. Lett. 61, 2445 (1988).

[6] D. Krökel, N. J. Halas, G. Giuliani, and D. Grischkowsky, Phys. Rev. Lett. 60, 29 (1988).

[7] E. Smirnov, C. E. Rüter, M. Stepić, D. Kip, and V. Shandarov, Phys. Rev. E 74, 065601 (2006).

[8] B. Denardo, W. Wright, S. Putterman, and A. Larraza, Phys. Rev. Lett. 64, 1518 (1990).

[9] A. Chabchoub, O. Kimmoun, H. Branger, N. Hoffmann, D. Proment, M. Onorato, and N. Akhmediev, Phys. Rev. Lett. 110, 124101 (2013).

[10] R. Heidemann, S. Zhdanov, R. Sütterlin, H. M. Thomas, and G. E. Morfill, Phys. Rev. Lett. 102, 135002 (2009).

[11] S. Burger, K. Bongs, S. Dettmer, W. Ertmer, K. Sengstock, A. Sanpera, G. V. Shlyapnikov, and M. Lewenstein, Phys. Rev. Lett. 83, 5198 (1999).

[12] C. Becker, S. Stellmer, P. Soltan-Panahi, S. Dorscher, M. Baumert, E.-M. Richter, J. Kronjager, K. Bongs, and K. Sengstock, Nat. Phys. 4, 496 (2008).

[13] Z. Dutton, M. Budde, C. Slowe, and L. V. Hau, Science 293, 663 (2001).

[14] B. P. Anderson, P. C. Haljan, C. A. Regal, D. L. Feder, L. A. Collins, C. W. Clark, and E. A. Cornell, Phys. Rev. Lett. 86, 2926 (2001).

[15] E. A. Kuznetsov and S. K. Turitsyn, Sov. Phys. JETP 67, 1583-1588 (1988).

[16] J. Brand and W. P. Reinhardt, Phys. Rev. A 65, 043612 (2002).

[17] P. Kevrekidis, G. Theocharis, D. Frantzeskakis, and A. Trombettoni, Phys. Rev. A 70, 023602 (2004).

[18] A. Muryshev, G. V. Shlyapnikov, W. Ertmer, K. Sengstock, and M. Lewenstein, Phys. Rev. Lett. 89, 110401 (2002).
[19] G. A. El and M. A. Hoefer, Physica D (in press) (2016), 10.1016/j.physd.2016.04.006.

[20] A. V. Gurevich and L. P. Pitaevskii, Sov. Phys. JETP 38, 291 (1974).

[21] A. V. Gurevich and A. L. Krylov, Sov. Phys. JETP 65, 944 (1987).

[22] M. Hoefer and B. Ilan, SIAM Mult. Mod. Sim. 10, 306 (2012).

[23] C. J. Pethick and H. Smith, Bose-Einstein Condensation in Dilute Gases, 2nd ed. (Cambridge University Press, 2008).

[24] L. D. Carr, C. W. Clark, and W. P. Reinhardt, Phys. Rev. A 62, 063610 (2000).

[25] S. Komineas and N. Papanicolaou, Phys. Rev. A 68, 043617 (2003).

[26] M. Ma, R. Carretero-González, P. Kevrekidis, D. Frantzeskakis, and B. Malomed, Phys. Rev. A 82, 023621 (2010).

[27] S. Middelkamp, P. Kevrekidis, D. Frantzeskakis, R. Carretero-González, and P. Schmelcher, Physica D 240, 1449 (2011).

[28] T. Aioi, T. Kadokura, T. Kishimoto, and H. Saito, Phys. Rev. X 1, 021003 (2011).

[29] T. P. Simula, Phys. Rev. A 84, 021603 (2011).

[30] C. Becker, K. Sengstock, P. Schmelcher, P. Kevrekidis, et al., New J. Phys. 15, 113028 (2013).

[31] A. Muñoz Mateo and J. Brand, Phys. Rev. Lett. 113, 255302 (2014).

[32] P. Scherpelz, K. Padavić, A. Rançon, A. Glatz, I. S. Aranson, and K. Levin, Phys. Rev. Lett. 113, 125301 (2014).

[33] M. J. Ku, W. Ji, B. Mukherjee, E. Guardado-Sanchez, L. W. Cheuk, T. Yefsah, and M. W. Zwierlein, Phys. Rev. Lett. 113, 065301 (2014).

[34] A. Bulgac, M. M. Forbes, M. M. Kelley, K. J. Roche, and G. Wlazłowski, Phys. Rev. Lett. 112, 025301 (2014).

[35] S. Donadello, S. Serafini, M. Tylutki, L. P. Pitaevskii, F. Dalfovo, G. Lamporesi, and G. Ferrari, Phys. Rev. Lett. 113, 065302 (2014).

[36] C. T. Kelley, Solving Nonlinear Equations with Newton's Method (Siam, 2003)

[37] Z. Wang and B. Hunt, Appl. Math. Comp. 16, 19 (1985). 